\title{
Perceptions of fatigue in patients with idiopathic pulmonary fibrosis or sarcoidosis
}

\author{
Ada E. M. Bloem ${ }^{1,2}$, Rémy L. M. Mostard ${ }^{3}$, Naomi Stoot ${ }^{3}$, Jan H. Vercoulen ${ }^{4}$, Jeannette B. Peters ${ }^{5}$, \\ Martijn A. Spruit ${ }^{6,7}$
}

${ }^{1}$ University of Applied Sciences Utrecht, Institute of Movement Studies, Faculty of Health Care, Utrecht, The Netherlands; ${ }^{2}$ ILD Centre of Excellence, Department of Pulmonology, St. Antonius Hospital, Nieuwegein, The Netherlands; ${ }^{3}$ Department of Respiratory Medicine, Zuyderland Medical Center Heerlen, Heerlen/Sittard-Geleen, The Netherlands; ${ }^{4}$ Department of Medical Psychology, Radboud University Medical Center, Radboud Institute for Health Sciences, Nijmegen, The Netherlands; ${ }^{5}$ Department of Pulmonary Diseases, Radboud University Medical Center, Radboud Institute for Health Sciences, Nijmegen, The Netherlands; ${ }^{6}$ Department of Research and Development, CIRO, Horn, The Netherlands; ${ }^{7}$ Department of Respiratory Medicine, Maastricht University Medical Center, NUTRIM School of Nutrition and Translational Research in Metabolism, Faculty of Health, Medicine and Life Science, Maastricht, The Netherlands

Contributions: (I) Conception and design: AEM Bloem, RLM Mostard, JH Vercoulen, MA Spruit; (II) Administrative support: AEM Bloem, N Stoot; (III) Provision of study materials or patients: RLM Mostard, N Stoot; (IV) Collection and assembly of data: AEM Bloem; (V) Data analysis and interpretation: AEM Bloem, RLM Mostard, JH Vercoulen, JB Peters, MA Spruit; (VI) Manuscript writing: All authors; (VII) Final approval of manuscript: All authors.

Correspondence to: Ada E. M. Bloem. Heidelberglaan 7, 3584 CS, Utrecht, The Netherlands. Email: aadje.bloem@hu.nl.

Background: Fatigue is highly prevalent in patients with idiopathic pulmonary fibrosis (IPF) or sarcoidosis. However, the difference in fatigue perceptions for these patients is unknown and this may be important to better understand what fatigue means to the individual patient.

Methods: This cross-sectional quantitative study aims to determine the different perceptions of fatigue as 'frustrating', 'exhausting', 'pleasant', 'frightening' using the Fatigue Quality List and to assess determinants related to these perceptions of fatigue. Beside the fatigue quality connotations, demographics, lung function, fatigue severity (Checklist Individual Strength subscale Fatigue), dyspnea (modified-Medical Research Council), fatigue catastrophizing (Fatigue Catastrophizing Scale), anxiety/depression (Hospital Anxiety and Depression Scale) and general health status (EuroQoL 5-dimension 5-level) were assessed.

Results: Mean frequency score of fatigue-related perceptions in patients with IPF was 3.4 points and in patients with sarcoidosis 4.0 points. Severely fatigued patients with IPF reported their fatigue less 'pleasant' significantly more often than patients without severe fatigue. Fatigue severity, dyspnea, catastrophizing and general health were significantly correlated with the negative connotation categories of the Fatigue Quality List in patients with IPF. Severely fatigued sarcoidosis patients reported their fatigue perceptions significantly more often as 'frustrating', 'exhausting', 'frightening' and less 'pleasant' than patients without severe fatigue. Moreover, in patients with sarcoidosis fatigue severity, dyspnea, catastrophizing and depression were significantly associated with all four categories of the Fatigue Quality List that describe the experienced fatigue $(\mathrm{P}<0.05)$.

Conclusions: The current findings of experiences of fatigue in patients with IPF or pulmonary sarcoidosis provide insights for professionals treating these patients. Although similarities were found in the several experiences of fatigue across non-severely and severely fatigued patients, differences were also evident and could be mapped for IPF and sarcoidosis.

Keywords: Idiopathic pulmonary fibrosis (IPF); sarcoidosis; fatigue; perceptions

Submitted Mar 17, 2021. Accepted for publication Jul 09, 2021.

doi: $10.21037 /$ jtd-21-462

View this article at: https://dx.doi.org/10.21037/jtd-21-462 


\section{Introduction}

Idiopathic pulmonary fibrosis (IPF) and sarcoidosis are diseases belonging to the interstitial lung diseases (ILDs). ILDs comprise a large group of respiratory disorders affecting the interstitium of the lungs $(1,2)$. Although ILDs include diseases with different pathophysiology and prognostics, most patients with ILD experience similar symptoms as shortness of breath, fatigue, dry cough and impaired exercise tolerance. It is known that fatigue is highly prevalent in patients with idiopathic pulmonary fibrosis (IPF) or sarcoidosis (1-6). To date, no data are available about the different perceptions of fatigue in these patients. This, however, may be important to create a better understanding of what fatigue means to the individual patient, and to determine whether or not it is a possible target for intervention.

The perception of fatigue can differ between individuals. For example, healthy individuals use adjectives such as normal, pleasant, relaxing, fulfilling and temporary to describe fatigue (7). Contrary, patients with different chronic conditions (i.e., chronic fatigue syndrome, neuromuscular diseases, pancreatitis, post-cancer fatigue, rheumatoid arthritis and chronic obstructive pulmonary disease) more often use negative adjectives such as frustrating, exhausting, upsetting and frightening (7-9).

The degree of fatigue severity may, at least partially, influence the perception of fatigue. Indeed, patients with severe fatigue describe their fatigue more often as frustrating or exhausting, whereas non-severely fatigued patients perceived their fatigue as normal $(7,8)$. Additionally, symptoms of breathlessness, anxiety/depression and catastrophic thoughts of fatigue may also have contributed to the different experiences of fatigue $(9,10)$. IPF and sarcoidosis are two different chronic pulmonary diseases in pathophysiological perspective, in which IPF is an fatal disease with progressive fibrosing of the lung with only some delay by antifibrotic medication and sarcoidosis is characterized by granulomatous inflammation with possible immunosuppressant therapy $(1,2)$. Severe fatigue was present $48 \%$ of patients with IPF and $69 \%$ in patients with sarcoidosis, but the differences in perceptions remained unclear.

To find out more about the perceptions of fatigue in patients with IPF or sarcoidosis, the aims of this study were: (I) to assess the different perceptions of fatigue in IPF or pulmonary sarcoidosis; (II) to evaluate the association between the different perceptions of fatigue and clinical parameters; and (III) to assess determinants related to general health.

We present the following article in accordance with the SURGE reporting checklist (available at https://dx.doi. org/10.21037/jtd-21-462).

\section{Methods}

\section{Study design and participants}

In the period between May 2018 and March 2019, patients (age $\geq 18$ years) with a confirmed diagnosis of IPF $(2,11)$ or pulmonary sarcoidosis $(12,13)$ were invited to participate in this cross-sectional clinical fatigue study (6). The study setting was at the outpatient clinic of the Department of Respiratory Medicine, Zuyderland Medical Centre Heerlen (The Netherlands). Patients were excluded if one of the following issues occurred: insufficient understanding of the Dutch language and/or inability to complete questionnaires due to cognitive impairment, or simultaneous participation in a potentially conflicting intervention study. Before this study (no funding) started, Medical Ethical Committee approval was granted (METCZ20180027) and registration with the Netherlands Trial Register (Code 7201) was established. The study was conducted in accordance with the Declaration of Helsinki (as revised in 2013).

The study protocol was in short: The chest physician (RM) invited eligible patients during the regular outpatient visit to participate in the study and he provided them with the written explanation. After that, patients met the nurse practitioner (NS) for complementary information of study participation and, if partaking, written informed consent; All participations were given a unique code (NS). Study participation involved completing the paper-andpencil questionnaires directly or at home after a reflection period; The one-time participation was on a voluntary basis. Data were collected from medical records (NS) to provide information on: age (years), gender (man/ woman), diagnosis, comorbidities, smoking pack-years (average packs smoked per day $\times$ duration of smoking, in years), medication, last available (within the preceding three months) spirometry (forced vital capacity, forced expiratory volume in one second; Liter, \% predicted), static lung volumes (total lung capacity, residual volume; Liter, \% predicted), and diffusing capacity for carbon 
monoxide (TLCO; \% predicted) (14).

\section{Measures}

\section{Experiences of fatigue}

The primary outcome measure was the difference in the experiences of fatigue. For the multidimensional perceptions of fatigue inventory the Fatigue Quality List (FQL) was used. The short form FQL consists of single choice questions (yes/no) for 18 adjectives (FQL-Adjectives) belonging to one of the FQL-categories 'frustrating', 'exhausting', 'pleasant' or 'frightening'. Patients were instructed to indicate which of the adjectives suited their experienced fatigue; multiple answers were allowed (7). Then the adjectives were divided into one of four fatigue description categories (FQL-Categories) with a score range of 0-100 points within each category. Adjectives belonging to the category frustrating (FQL-Frustrating) are discouraging, incessant, annoying, persistent and frustrating. Adjectives belonging to the category exhausting (FQL-Exhausting) are exhausting, wearisome, extreme and unbearable. In the category pleasant (FQL-Pleasant) are temporary, relaxing, fulfilling, normal and pleasant. And in the category frightening (FQL-Frightening) are upsetting, frightening, inexplicable and insuperable (7).

\section{Demographic characteristics}

Participants self-reported the following demographic data on partner (yes/no), living situation (alone/cohabiting), education ("low education level" meaning maximally preparatory school and "high education level" meaning minimally secondary vocational education), height (m), weight (kg), hospitalization last year (yes/no), working situation last 2 years (yes/no), psychological support (yes/ no) and smoking (former/current, never).

\section{Self-reported questionnaires}

Fatigue severity was assessed by the Checklist Individual Strength subscale fatigue (CIS-Fatigue). The subscale CISFatigue (eight items) investigates the patients' severity of fatigue of the previous two weeks $(15,16)$. Each item was scored on a seven-point Likert scale with a total item score range of $8-56$ points. A score of points $\leq 26$ indicates normal fatigue, between 27-35 moderate fatigue (called together non-severe fatigue $<36$ points), and a score of $\geq 36$ indicates severe fatigue $(17,18)$.

Activity-related dyspnea was measured with the modified medical research council scale for dyspnea
(mMRC). The severity of dyspnea is indicated on a 5 -point scale ( $0-4$ score; higher level reflects more activity limitation due to dyspnea) (19).

To investigate catastrophizing thoughts related to fatigue the Fatigue Catastrophizing Scale (FCS) was used. The FCS was adapted from the pain catastrophizing scale (PCS) by replacing the term "pain" with "fatigue" where relevant (20). This scale consists of 13 items measuring the relation of catastrophizing to fatigue on a 5 -point scale from 0 (not at all) to 4 (always), with a total maximum of 52 points. Higher scores are indicative for more fatigue catastrophizing (21).

The Hospital Anxiety and Depression Scale (HADS) is a self-reporting instrument to estimate emotional distress, consisting of two subscales for anxiety (HADS-Anxiety) and depression (HADS-Depression) in somatically ill patients $(22,23)$. Total scores for each subscale range from 0 to 21 points, categorized as: normal/mild ( $0-10$ points) and moderate/severe (11-21 points, meaning a clinically significant case of anxiety or depression) (22).

The patients' self-rated general health (score of today) was recorded with the visual analog scale of the European Quality of Life-5 Dimensions (EQ-5D-5L VAS) and the 5-dimension list (mobility, self-care, usual activities, pain/ discomfort, anxiety/depression) (24). The vertically situated VAS had labelled endpoints from 0 (worst imaginable health) to 100 points (best imaginable health). The 5 -dimension list results were converted into an index value (EQ-5D-5L index value) (25).

\section{Statistical analyses}

Data were presented with appropriate measures of central tendency and dispersion, as mean \pm standard deviation (SD), median and interquartile range, or frequencies and proportions. Numerical data were tested for normality by Shapiro-Wilkinson test, a mean-median ratio, SD-mean ratio, and judging the histogram (26). Differences between groups for continuous data were analyzed by an unpaired $t$-test or the non-parametric pendant (Mann-Whitney $\mathrm{U}$ test) where appropriate. Categorical data were analyzed with the Chi-square or Fisher Exact test. A P value of $\leq 0.05$ was considered as statistically significant. Univariate differences between non-severely (mild/moderate fatigue) and severely fatigued patients were tested with independent $t$-tests, Mann-Whitney U-tests and chi-square-tests were performed as appropriate. Pearson's $r$ or Spearman's rho were used to study correlations between the continuous data FQL-Categories or EQ-5D-5L (VAS or index value) 
and patient characteristics or psychosocial factors. In case of missing values, cases were excluded pairwise. The range for what constitutes a weak, moderately strong, strong, or very strong correlation was respectively $0.1 \leq \mathrm{r}<0.3,0.3 \leq \mathrm{r}<0.5$, $0.5 \leq \mathrm{r}<0.7$, and $0.7 \leq \mathrm{r}<1.0[45,46]$ (level of significance $\mathrm{P}<0.05)$. Stepwise multiple linear regression with backward selection procedure was performed to investigate the associations between the EQ-5D-5L VAS or EQ-5D$5 \mathrm{~L}$ index value and independent in univariate analysis significant variables $(\mathrm{P}<0.05)$. In case of multicollinearity, variables were identified [variance inflation factor (VIF) $>5$ ] and removed from the model (27). IBM SPSS Statistics (Version 25) was used for statistical analysis.

\section{Results}

\section{General characteristics}

Of the 170 patients invited to participate in this fatigue study, 61 patients with IPF responded (92\% response rate) and 60 patients with sarcoidosis (58\% response rate). General characteristics of the patients with IPF or sarcoidosis are shown in Table 1. Most patients with IPF were elderly men, with a low education level, a smoking history and a slightly increased body mass index (BMI). They had an impaired lung function, most of these patients were diagnosed with one or more comorbidities, and $87 \%$ used anti-fibrotic medication. Severe fatigue was prevalent in $48 \%$ of the patients with IPF and $51 \%$ had more severe scores on activity-related dyspnea $(\mathrm{mMRC} \geq 2)$. Moreover, moderate/severe anxiety or depression was present in $9 \%$ of the patients with IPF, patients had a mild degree of catastrophic thoughts related to fatigue, and 52\% scored VAS general health at 60 points or less (data not shown).

The patients with sarcoidosis were middle aged and had an increased mean BMI. Patients mainly used medication such as immuno-suppressants (39\%) and divers medication for lung conditions (56\%). 69\% of patients with sarcoidosis were severely fatigued and $36 \%$ were more impaired in walking due to dyspnea $(\mathrm{mMRC} \geq 2)$. Also, moderate/severe anxiety and depression was reported by $12 \%$ and $8 \%$ of the patients with sarcoidosis. They had a mildly elevated score of catastrophizing, and $46 \%$ selfrated general health at 60 points or less (data not shown).

\section{Perceptions of fatigue in patients with IPF}

Patients were allowed to report as many adjectives for fatigue as they found appropriate. In patients with IPF the mean score was 3.4 adjectives (3.2 SD). The distribution of the frequency of adjectives is depicted in Figure $1 A$. The mean number of adjectives was similar between IPF patients with or without severe fatigue (4 versus 3 respectively, $\mathrm{P}>0.05)$. IPF patients with severe fatigue reported significantly more adjectives as upsetting, incessant, wearisome and persistent than the non-fatigued patients. Not one patient stated fatigue as normal. With respect to the categories of the FQL, severely fatigued patients with IPF only scored significantly less on FQL-Pleasant than non-severe fatigued patients (Table 2).

\section{Perceptions of fatigue in patients with sarcoidosis}

The mean of the sum of adjectives reported by patients with sarcoidosis was 4.0 (3.0 SD). Severely fatigued patients with sarcoidosis used a higher number of adjectives to describe their experience of fatigue than the non-severe fatigued patients: 5 versus 2 adjectives, respectively $(\mathrm{P}<0.05$; Table 3, Figure $1 B$ ). The severely fatigued patients more often reported the following adjectives of fatigue: discouraging, temporary, exhausting, incessant, wearisome, annoying, extreme, persistent, frustrating, inexplicable and normal (Table 3). With respect to the categories of the FQL, patients with severe fatigue significantly scored higher on FQL-Frustrating, FQL-Exhausting and FQL-Frightening, and significantly less on the FQL-Pleasant than patients with non-severe fatigue $(\mathrm{P}<0.05$; Table 2$)$.

\section{Perceptions of fatigue in patients with IPF or sarcoidosis with severe fatigue}

Severely fatigued patients with IPF or sarcoidosis generally used the same percentage of adjectives to express their fatigue experience. However, the adjectives exhausting and frustrating were reported by a significant higher number of severely fatigued patients with sarcoidosis as compared to severely fatigued patients with IPF. Moreover, there were no significant differences between both groups with respect to the categories FQL-Frustrating, FQL-Exhausting, FQLPleasant and FQL-Frightening, and the highest scores were seen in the category FQL-Frustrating in both patient groups (IPF 34 points, sarcoidosis 48 points).

\section{Univariate correlations of perceptions of fatigue}

Table 4 gives an overview of all significant correlations 
Table 1 General characteristics of patients with interstitial lung disease, idiopathic pulmonary fibrosis (IPF) or sarcoidosis

\begin{tabular}{|c|c|c|}
\hline Variables & Patients with IPF $(n=61)$ & Patients with Sarcoidosis $(n=60)$ \\
\hline Gender, male, n (\%) & $47(77.0)$ & $29(48.3)$ \\
\hline Age, years [IQR] & $73.0[70-78]$ & $53.5[46.3-62.0]$ \\
\hline Partner, n (\%) & $45(73.8)$ & $44(73.3)$ \\
\hline Education, low <secondary level, $\mathrm{n}(\%)^{\mathrm{b}}$ & $30(50.8)$ & $13(21.7)$ \\
\hline Diagnosis time, $\leq 1$ year, $\mathrm{n}(\%)^{\mathrm{b}}$ & $21(35.0)$ & $16(27.1)$ \\
\hline Hospitalization, $\leq 1$ year, $\mathrm{n}(\%)^{\mathrm{a}}$ & $14(23.0)$ & $10(16.9)$ \\
\hline Work, last 2 years, n (\%) & $12(19.7)$ & $37(61.7)$ \\
\hline Pack-years $^{+}$, smoking current/former & $19.5 \pm 19.4$ & $10.6 \pm 13.9$ \\
\hline \multicolumn{3}{|l|}{ Physiological } \\
\hline $\operatorname{BMI}\left(\mathrm{kg} / \mathrm{m}^{2}\right)^{\mathrm{a}}$ & $27.5 \pm 4.1$ & $27.6 \pm 4.2$ \\
\hline TLC (liter) $)^{\mathrm{h}}$ & $4.6 \pm 1.1$ & $6.0 \pm 1.3$ \\
\hline TLC (\% predicted $)^{i}$ & $73.3 \pm 14.1$ & $98.5 \pm 18.5$ \\
\hline RV (liter) $)^{g}$ & $1.6 \pm 0.4$ & $2.0 \pm 0.5$ \\
\hline RV (\% predicted) ${ }^{i}$ & $64.8 \pm 14.2$ & $97.9 \pm 25.7$ \\
\hline FVC (liter) & $2.9 \pm 0.8$ & $3.9 \pm 1.1$ \\
\hline Comorbidity $\leq 1, \mathrm{n}(\%)^{\mathrm{b}}$ & $45(75)$ & $30(51)$ \\
\hline \multicolumn{3}{|l|}{ Medication } \\
\hline IPF - antifibrotic, n (\%) & $53(86.9)$ & $0(0.0)$ \\
\hline Immunosuppressant incl. prednisone (corticosteroids), $\mathrm{n}(\%)^{\mathrm{c}}$ & $5(8.5)$ & $23(39.0)$ \\
\hline Heart rate-lowering medication, $\mathrm{n}(\%)^{\mathrm{c}}$ & $16(27.1)$ & $7(11.9)$ \\
\hline Antidepressant medication, $\mathrm{n}(\%)^{\mathrm{c}}$ & $5(8.5)$ & $1(1.7)$ \\
\hline Antihypertensive medication, $\mathrm{n}(\%)^{\mathrm{c}}$ & $26(44.1)$ & $11(18.6)$ \\
\hline Medication for pulmonary conditions, $\mathrm{n}(\%)^{c}$ & $7(11.9)$ & $33(55.9)$ \\
\hline \multicolumn{3}{|l|}{ Fatigue } \\
\hline CIS-Fatigue $^{1}(p, 8-56)^{d}$ & $34.1 \pm 11.2$ & $40.0 \pm 12.3$ \\
\hline CIS, Severe $>35$ points, $n(\%)^{d}$ & $28(47.5)$ & $40(69.0)$ \\
\hline
\end{tabular}

Table 1 (continued) 
Table 1 (continued)

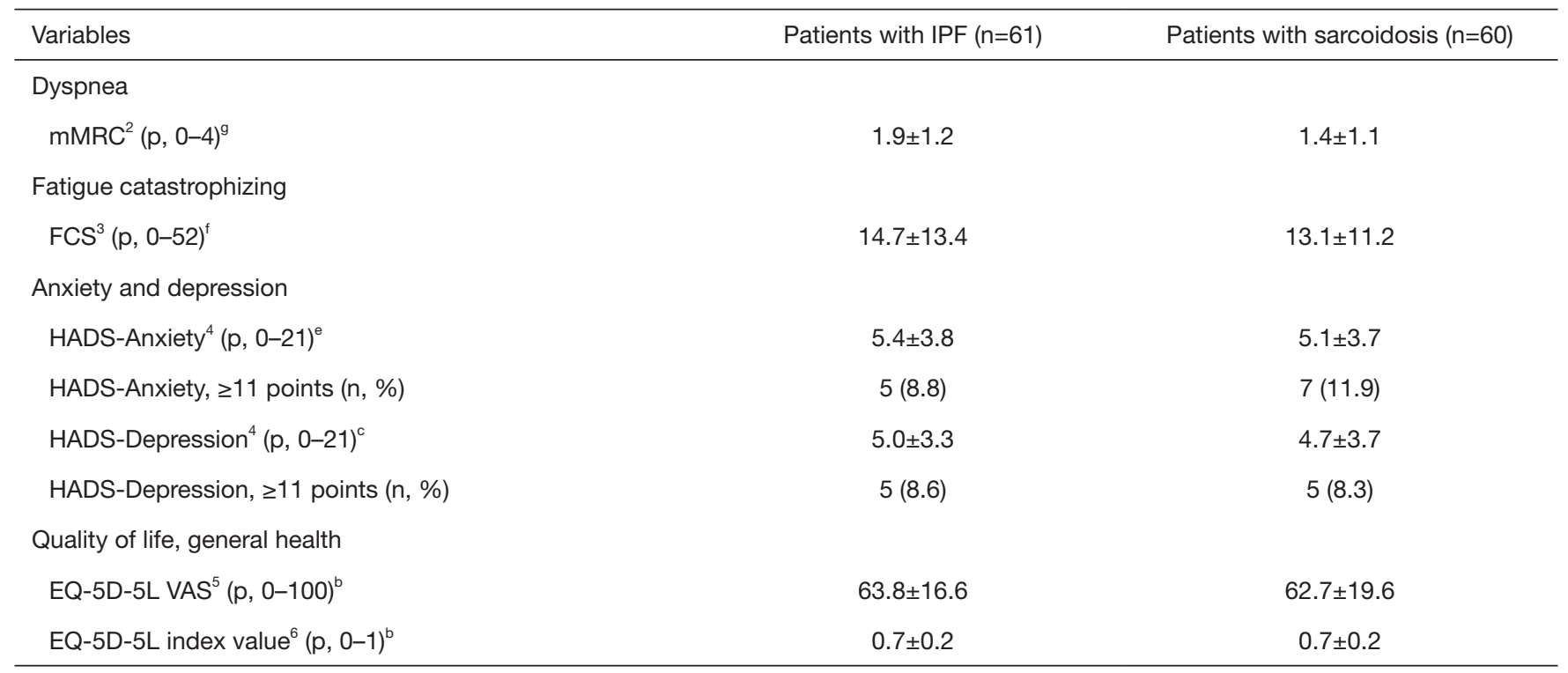

Data are presented as mean $\pm \mathrm{SD}$, median $[\mathrm{IQR}]$ or $\mathrm{n}(\%) .{ }^{+}$Pack-year, number of years smoking $\mathrm{x}$ average number of cigarettes smoked per day/20. Alphabetic characters in superscript indicates a sample size deviant from $n=121$, in the order: ${ }^{a} n=120,{ }^{b} n=119,{ }^{c} n=118,{ }^{d}$ $n=117,{ }^{e} n=116,{ }^{f} n=115,{ }^{9} n=114,{ }^{h} n=113,{ }^{i} n=112,{ }^{j} n=52 .{ }^{1}$, CIS-Fatigue scores range from 8 to 56 ; high scores indicate more fatigue; ${ }^{2}$, mMRC score range from 0 to 4 ; high scores indicate more activity related dyspnea; ${ }^{3}$, FCS score range from 0 to 52 ; high scores indicate more catastrophizing of fatigue; ${ }^{4}$, HADS-Anxiety or ${ }^{4} \mathrm{HADS}$-Depression score range from 0 to 21 ; high scores indicate more experience of anxiety or depression; ${ }^{5}$, EQ-5D-5L VAS score range from 0 to 100; high score indicate better general health (today); ${ }^{6}$, EQ5D-5L index value score range from 0 to 1; score 1 mean full health. BMI, body mass index; CIS-Fatigue, Checklist Individual Strength subscale Fatigue; EQ-5D-5L, EuroQol, 5 dimensions, 5 levels (standardised measure of health status); FCS, Fatigue Catastrophizing Scale; FEV1, forced expiratory volume in one second; FVC, forced vital capacity; HADS-Anxiety: hospital anxiety and depression scale subscale anxiety; HADS-Depression: hospital anxiety and depression scale subscale depression; IPF, idiopathic pulmonary fibrosis; IQR, interquartile range; $\mathrm{mMRC}$, modified Medical Research Council dyspnea scale; $p$, points; RV, residual volume; RV/TLC, residual volume to total lung capacity; TLC, total lung capacity; TLCO, transfer factor of the lung for carbon monoxide; VAS, Visual Analog Scale.
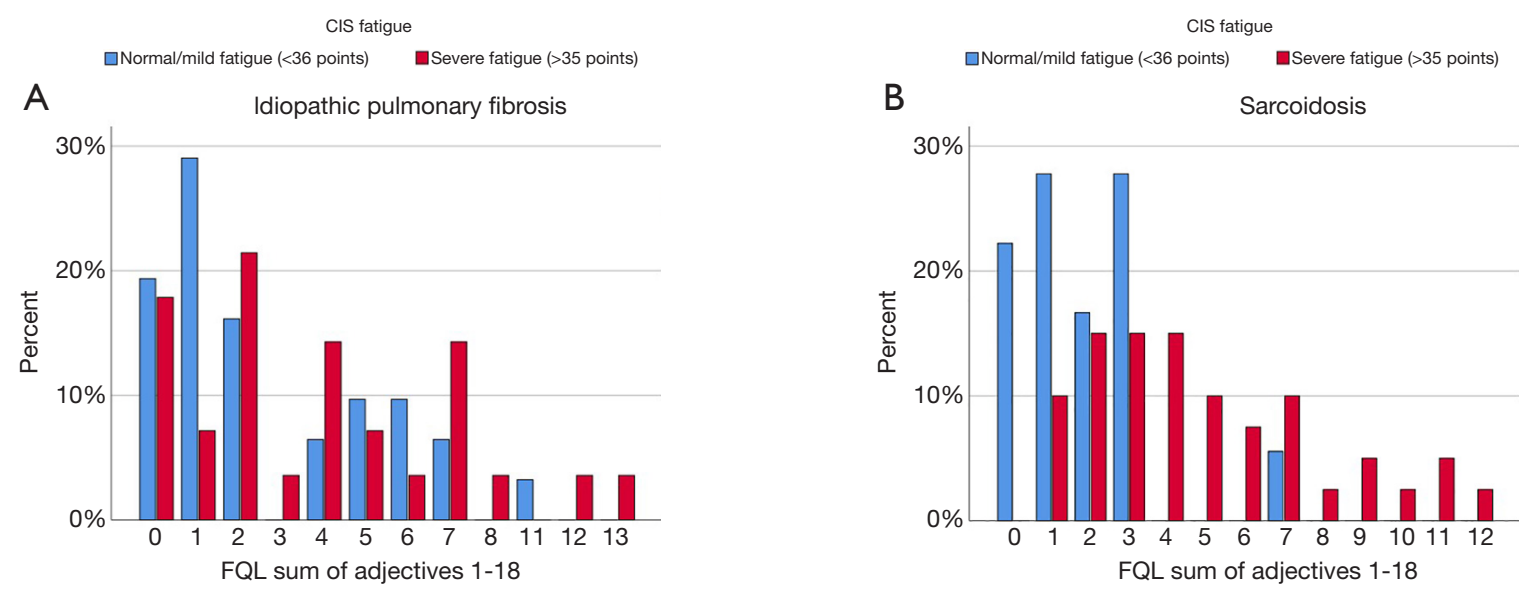

Figure 1 Frequency of adjectives for fatigue stratified for fatigue severity in patients with idiopathic pulmonary fibrosis or sarcoidosis. (A) Frequency of adjectives for fatigue stratified for fatigue severity in patients with idiopathic pulmonary fibrosis; (B) frequency of adjectives for fatigue stratified for fatigue severity in patients with sarcoidosis. CIS Fatigue, Checklist Individual Strength subscale Fatigue; FQL, Fatigue Quality List. 
Table 2 Categories perceptions of fatigue in patients with idiopathic pulmonary fibrosis or sarcoidosis, stratified for fatigue severity

\begin{tabular}{|c|c|c|c|c|c|c|c|c|c|}
\hline \multirow{2}{*}{ Categories } & \multirow{2}{*}{ IPF $(n=61)$} & \multicolumn{3}{|c|}{ Fatigue severity in patients with IPF } & \multirow{2}{*}{$\begin{array}{l}\text { Sarcoidosis } \\
\quad(n=60)\end{array}$} & \multicolumn{3}{|c|}{$\begin{array}{l}\text { Fatigue severity in patients with } \\
\text { sarcoidosis }\end{array}$} & \multirow{2}{*}{$\begin{array}{c}\begin{array}{c}\text { IPF-sarcoidosis, } \\
\text { severe fatigue }\end{array} \\
\text { P value }\end{array}$} \\
\hline & & $\begin{array}{c}\text { Non-severe } \\
\text { fatigue }(n=31)\end{array}$ & $\begin{array}{l}\text { Severe fatigue } \\
\qquad(n=28)\end{array}$ & $P$ value & & $\begin{array}{l}\text { Non-severe } \\
\text { fatigue }(n=18)\end{array}$ & $\begin{array}{l}\text { Severe fatigue } \\
\qquad(N=40)\end{array}$ & $P$ value & \\
\hline $\begin{array}{l}\text { Frustrating } \\
(p, 0-100)\end{array}$ & $26.2 \pm 30.9$ & $18.1 \pm 24.4$ & $33.6 \pm 34.9$ & NS & $36.3 \pm 32.6$ & $7.8 \pm 14.0$ & $48 \pm 30.0$ & $<0.05$ & NS \\
\hline $\begin{array}{l}\text { Pleasant } \\
(p, 0-100)\end{array}$ & $12.1 \pm 19.4$ & $17.4 \pm 24.1$ & $7.1 \pm 11.2$ & $<0.05$ & $10.7 \pm 15.4$ & $20.0 \pm 18.2$ & $7.0 \pm 12.4$ & $<0.05$ & NS \\
\hline $\begin{array}{l}\text { Frightening } \\
(\mathrm{p}, 0-100)\end{array}$ & $18.4 \pm 28.1$ & $13.7 \pm 27.3$ & $24.1 \pm 29.3$ & NS & $17.5 \pm 25.3$ & $6.9 \pm 18.8$ & $21.3 \pm 26.9$ & $<0.05$ & NS \\
\hline
\end{tabular}

Data are presented as mean $\pm \mathrm{SD}$; $\mathrm{P}$ value (Student's $t$-test) in bold indicates a significant difference $<0.05$; Fatigue severity is based on the Checklist Individual Strength subscale Fatigue (CIS-Fatigue): 'Non-severe Fatigue' CIS-Fat <36 points, 'Severe Fatigue' CIS-Fat $\geq 36$ points. In case of missing data of CIS-Fatigue cases were excluded analysis by analysis. CIS-Fatigue, Checklist Individual Strength subscale Fatigue; IPF, idiopathic pulmonary fibrosis; NS, not significant; p, points. FQL scores range from 0 to 100; higher scores in each category indicate fatigue is frustrating, exhausting, pleasant, or frightening to a higher degree.

$(\mathrm{P}<0.05)$ between the four FQL-Categories and fatigue severity (CIS-Fatigue), dyspnea (mMRC), fatigue catastrophizing (FCS), anxiety (HADS-Anxiety), depression (HADS-Depression) and general health status (EQ-5D-5L VAS). In patients with IPF catastrophizing fatigue correlated strongly $(0.5 \leq \rho<0.7)$ with the three categories of the FQL representing negative adjectives and not one of these concepts correlated with the experience of pleasant fatigue. In patients with sarcoidosis strong correlations were found between FQL-Frustrating and fatigue severity, catastrophizing, depression and general health, and in FQL-Exhausting the same traits supplemented with dyspnea and anxiety. FQL-Pleasant was strongly correlated with general health and FQLFrightening with catastrophizing (Table 4).

\section{Determinants of general bealth}

Table 5 shows the associations for general health (EQ-5D5L VAS and EQ-5D-5L index value) with fatigue severity (CIS-Fatigue), experience of fatigue (FQL-categories) and other clinical parameters. In patients with IPF moderately strong correlations with general health (EQ-5D-5L VAS or index value) were found with the FQL-Frustrating, FQL-Exhausting and FQL-Frightening, but no significant correlation with FQL-Pleasant. In patients with sarcoidosis general health (EQ-5D-5L VAS or index value) showed strong correlations with FQL-frustrating, FQL-Exhausting and FQL-Pleasant, and moderately strong correlation of the EQ-5D-5L index value with FQL-Frightening. The regression equations with the dependent variables EQ5D-5L VAS or index value are presented in Table 6. The explained variance (R2) of the stepwise multiple regression models for patients with IPF $v s$. sarcoidosis was for EQ-5D5L VAS $58 \%$ vs. $59 \%$ and for EQ-5D-5L index value $75 \%$ vs. $56 \%$.

\section{Discussion}

This study shows that there are extensive variations in perceptions of fatigue in patients with IPF or sarcoidosis, in addition to the severity of fatigue. Patients expressed their perceived fatigue in a different number of adjectives, ranging from 0 to 13 adjectives. Also, severely fatigued patients with IPF perceived their fatigue (categories FQL) less as pleasant than non-severely fatigued IPF patients. Severely fatigued patients with sarcoidosis perceived their fatigue as more frustrating, exhausting, frightening and less pleasant compared to non-severely fatigued patients. Although a third of the patients without severe fatigue reported their fatigue as normal and temporary, some of these non-severely fatigued patients also had negative associations with fatigue, such as exhausting (14\%), frightening (14\%), annoying (18\%) and frustrating (29\%). The findings in this study for negative connotations of fatigue are in line with other chronic diseases (7-9). 
Table 3 Perceptions of fatigue in patients with idiopathic pulmonary fibrosis or sarcoidosis, stratified for fatigue severity

\begin{tabular}{|c|c|c|c|c|c|c|c|c|c|}
\hline \multirow[b]{2}{*}{ Fatigue Quality List } & \multirow[b]{2}{*}{$\operatorname{IPF}(n=61)$} & \multicolumn{3}{|c|}{$\begin{array}{l}\text { Fatigue severity in patients with } \\
\qquad \text { IPF }\end{array}$} & \multirow{2}{*}{$\begin{array}{l}\text { Sarcoidosis } \\
\qquad(\mathrm{n}=60)\end{array}$} & \multicolumn{3}{|c|}{$\begin{array}{c}\text { Fatigue severity in patients with } \\
\text { sarcoidosis }\end{array}$} & \multirow{2}{*}{$\begin{array}{c}\text { IPF - sarcoidosis, } \\
\text { severe fatigue } \\
\text { P value }\end{array}$} \\
\hline & & $\begin{array}{c}\text { Non-severe } \\
\text { fatigue } \\
(n=31)\end{array}$ & $\begin{array}{c}\text { Severe } \\
\text { fatigue } \\
(n=28)\end{array}$ & $P$ value & & $\begin{array}{c}\text { Non-severe } \\
\text { fatigue } \\
(n=18)\end{array}$ & $\begin{array}{l}\text { Severe } \\
\text { fatigue } \\
(N=40)\end{array}$ & $P$ value & \\
\hline Upsetting, n (\%) & $14(23.0)$ & $4(12.9)$ & $10(35.7)$ & $<0.05^{\star}$ & $11(18.3)$ & $2(11.1)$ & $9(22.5)$ & $N S^{\star *}$ & $\mathrm{NS}^{*}$ \\
\hline Temporary, n (\%) & $16(26.2)$ & $10(32.3)$ & $6(21.4)$ & $\mathrm{NS}^{*}$ & $18(30.0)$ & $9(50.0)$ & $9(22.5)$ & $<0.05^{\star}$ & $\mathrm{NS}^{*}$ \\
\hline Exhausting, n (\%) & $15(24.6)$ & $6(19.4)$ & $8(28.6)$ & $\mathrm{NS}^{*}$ & $22(36.7)$ & $1(5.6)$ & $21(52.5)$ & $<0.05^{\star}$ & NS $(P=0.05)^{*}$ \\
\hline Incessant, n (\%) & $12(19.7)$ & $3(9.7)$ & $9(32.1)$ & $<0.05^{\star}$ & $20(33.3)$ & $1(5.6)$ & $18(45.0)$ & $<0.05^{\star}$ & $\mathrm{NS}^{*}$ \\
\hline Wearisome, n (\%) & $17(27.9)$ & 4 (12.9) & $12(42.9)$ & $<0.05^{\star}$ & $15(25.0)$ & $1(5.6)$ & $14(35.0)$ & $<0.05^{\star \star}$ & $\mathrm{NS}^{*}$ \\
\hline Extreme, n (\%) & 7 (11.5) & $2(6.5)$ & $4(14.3)$ & $N S^{\star *}$ & $10(16.7)$ & $0(0.0)$ & $10(25.0)$ & $<0.05^{\star \star}$ & $\mathrm{NS}^{*}$ \\
\hline Persistent, n (\%) & $11(18.0)$ & $2(6.5)$ & $8(28.6)$ & $<0.05^{\star \star}$ & $21(35.0)$ & $1(5.6)$ & $19(47.5)$ & $<0.05^{\star}$ & $\mathrm{NS}^{*}$ \\
\hline Frustrating, n (\%) & $24(39.3)$ & $11(35.5)$ & $11(39.3)$ & $\mathrm{NS}^{*}$ & $33(55.0)$ & $3(16.7)$ & $28(70.0)$ & $<0.05^{\star}$ & $<0.05^{*}$ \\
\hline Relaxing, n (\%) & $5(8.2)$ & $2(6.5)$ & $3(10.7)$ & $N S^{\star \star}$ & $1(1.7)$ & $0(0.0)$ & $1(2.5)$ & $\mathrm{NS}^{\star *}$ & $\mathrm{NS}^{\star \star}$ \\
\hline Inexplicable, n (\%) & $10(16.4)$ & $3(9.7)$ & $6(21.4)$ & $N S^{\star *}$ & $15(25.0)$ & $1(5.6)$ & $13(32.5)$ & $<0.05^{\star}$ & $\mathrm{NS}^{*}$ \\
\hline Fulfilling, n (\%) & $2(3.3)$ & $2(6.5)$ & $0(0.0)$ & $N S^{\star \star}$ & $3(5.0)$ & $2(11.1)$ & $1(2.5)$ & $\mathrm{NS}^{* \star}$ & $\mathrm{NS}^{* \star}$ \\
\hline Insuperable, n (\%) & $10(16.4)$ & $4(12.9)$ & $6(21.4)$ & $N S^{* *}$ & $8(13.3)$ & $1(5.6)$ & $6(15.0)$ & $N S^{\star \star}$ & $\mathrm{NS}^{*}$ \\
\hline Unbearable, n (\%) & $4(6.6)$ & $1(3.2)$ & $2(7.1)$ & $N S^{\star *}$ & 7 (11.7) & $1(5.6)$ & $5(12.5)$ & $\mathrm{NS}^{\star \star}$ & $N S^{\star *}$ \\
\hline
\end{tabular}

Data are presented as $\mathrm{n}(\%)$ or mean $\pm \mathrm{SD}, \mathrm{P}$ value in bold indicates a significant difference $<0.05 .{ }^{*}$, Pearson Chi-Square test; **, Fisher's Exact Test; ", Student's T-test. Fatigue severity is based on the Checklist Individual Strength subscale Fatigue: 'Non-severe Fatigue' CIS-Fatigue $<36$ points, 'Severe Fatigue' CIS-Fatigue $\geq 36$ points. In case of missing data of CIS-Fatigue cases were excluded analysis by analysis. CIS-Fatigue, Checklist Individual Strength subscale Fatigue; IPF, idiopathic pulmonary fibrosis; ns, not significant; $p$, points.

Negative connotations were mentioned in both diseases IPF or sarcoidosis and most prevalent were frustrating (39\% IPF, 55\% sarcoidosis), followed by exhausting (IPF 25\%, sarcoidosis 37\%) and annoying (IPF 33\%, sarcoidosis $33 \%)$. In severely fatigued patients with IPF or sarcoidosis these percentages of negative connotations were even higher and also other negative connotations were substantially prevalent $(>30 \%)$ as wearisome, incessant, upsetting, discouraging and inexplicable. Catastrophizing thoughts about fatigue are associated with higher rates of experiencing fatigue as frustrating, exhausting, frightening and less rates of pleasant. So, for a better understanding of fatigue it is necessary to look at the severity of the fatigue on the one hand, and the perception of fatigue on the other hand. Moreover, patients without severe fatigue might experience negative associations concerning fatigue and patients with severe fatigue might not come up with very negative terms. In treatment of patients, it is useful to know how people view their fatigue, to connect with the perception of the patient and thus to find out what is desirable to change their cognitions and/or behavior. Treatment of fatigue can 
Table 4 Correlations of the categories of the FQL with fatigue, dyspnea, catastrophizing, anxiety, depression and general health in patients with idiopathic pulmonary fibrosis or sarcoidosis

\begin{tabular}{|c|c|c|c|c|c|c|c|c|}
\hline & \multicolumn{2}{|c|}{ FQL-Frustrating } & \multicolumn{2}{|c|}{ FQL-Exhausting } & \multicolumn{2}{|c|}{ FQL-Pleasant } & \multicolumn{2}{|c|}{ FQL-Frightening } \\
\hline \multicolumn{9}{|l|}{ Idiopathic pulmonary fibrosis } \\
\hline Fatigue (CIS-Fatigue) & 0.322 & $<0.05$ & 0.258 & $<0.05$ & -0.231 & NS & 0.351 & $<0.01$ \\
\hline Dyspnea (mMRC) & 0.350 & $<0.01$ & 0.343 & $<0.01$ & 0.235 & NS & 0.445 & $<0.01$ \\
\hline Anxiety (HADS-Anxiety) & 0.228 & NS & 0.252 & NS & -0.054 & NS & 0.466 & $<0.01$ \\
\hline Depression (HADS-Depression) & 0.172 & NS & 0.195 & NS & 0.141 & NS & 0.438 & $<0.01$ \\
\hline General health (EQ-5D-5L VAS) & -0.355 & $<0.01$ & -0.369 & $<0.01$ & -0.021 & NS & -0.350 & $<0.01$ \\
\hline \multicolumn{9}{|l|}{ Sarcoidosis } \\
\hline Fatigue catastrophizing (FCS) & 0.608 & $<0.01$ & 0.643 & $<0.01$ & -0.486 & $<0.01$ & 0.650 & $<0.01$ \\
\hline Anxiety (HADS-Anxiety) & 0.250 & NS & 0.530 & $<0.01$ & -0.237 & NS & 0.484 & $<0.01$ \\
\hline Depression (HADS-Depression) & 0.531 & $<0.01$ & 0.584 & $<0.01$ & -0.389 & $<0.01$ & 0.421 & $<0.01$ \\
\hline General health (EQ-5D-5L VAS) & -0.525 & $<0.01$ & -0.531 & $<0.01$ & 0.537 & $<0.01$ & -0.169 & NS \\
\hline
\end{tabular}

P, statistically significant; $\rho$, Spearman rank correlation coefficient. CIS-Fatigue, Checklist Individual Strength subscale Fatigue; EQ-5D5L, EuroQol, 5 dimensions, 5 levels (standardised measure of health status); FCS, Fatigue Catastrophizing Scale; FQL, Fatigue Quality Scale; HADS-Anxiety, Hospital Anxiety and Depression Scale subscale Anxiety; HADS-Depression, Hospital Anxiety and Depression Scale subscale Depression; mMRC, modified Medical Research Council-Dyspnea; VAS, Visual Analogue Scale.

be recommended in many ways and one of the treatment options is cognitive behavioral therapy (CBT), a type of psychotherapy. CBT aims to influence cognitions and behaviors that are related to the experienced problems $(28,29)$. CBT could be an intervention for reducing fatigue in patients with sarcoidosis or IPF, as is already seen in patients with sarcoidosis (30,31), cancer (32), chronic fatigue syndrome (33) and multiple sclerosis (34). CBT also aims at changing the patients' attitude towards fatigue and in that way changing the experience of fatigue. Patients with chronic conditions with more complex problematic conditions might be referred to pulmonary rehabilitation. In pulmonary rehabilitation, cognitive behavioral therapy is already integrated and considered for improving patients' physical, psychological and quality of life $(35,36)$. In addition to this known information, this study emphasizes the importance of taking into account the patients' different perceptions of fatigue in the rehabilitation of the patients. Further research is needed to investigate the most targeted approach to reduce negative experiences of fatigue.
It has been established that fatigue and depression are highly correlated and depression symptomatology and prevalence are significantly increased in fatigued individuals (37). In patients with sarcoidosis the HADSdepression score is significantly related to negative expressions of fatigue, but, in patients with IPF only to frightening. In the current study severe fatigue was present in $48 \%$ of the patients with IPF and in $69 \%$ of the patients with sarcoidosis. The depression rate of moderate/severe level was present in $9 \%$ of the patients with IPF and in $8 \%$ of the patients with sarcoidosis. These data support that fatigue is a common symptom in many physical and psychological disorders, such as depression. But that doesn't mean that fatigue and depression are the same phenomenon. In other words, a fatigued patient does not necessarily have to be depressed.

The symptoms fatigue and sleepiness are often used intermingled, but the symptom excessive daytime sleepiness (ESS) describes the probability to fall asleep in various situations at daytime (38). This study and a recent study 
Table 5 Correlations of general health (EQ-5D-5L-VAS and EQ-5D-5L-Index Value) in patients with idiopathic pulmonary fibrosis or sarcoidosis

\begin{tabular}{|c|c|c|c|c|c|c|c|c|}
\hline & \multicolumn{8}{|c|}{ General health } \\
\hline & \multicolumn{2}{|c|}{ EQ-5D-5L VAS } & \multicolumn{2}{|c|}{ EQ-5D-5L IV } & \multicolumn{2}{|c|}{ EQ-5D-5L VAS } & \multicolumn{2}{|c|}{ EQ-5D-5L IV } \\
\hline & $\rho$ & $\mathrm{P}$ & $\rho$ & $\mathrm{P}$ & $\rho$ & $\mathrm{P}$ & $\rho$ & $\mathrm{P}$ \\
\hline Fatigue severity (CIS-Fatigue) & -0.529 & $<0.01$ & -0.414 & $<0.01$ & -0.710 & $<0.01$ & -0.577 & $<0.01$ \\
\hline FQL-Exhausting category & -0.369 & $<0.01$ & -0.377 & $<0.01$ & -0.531 & $<0.01$ & -0.703 & $<0.01$ \\
\hline FQL-Pleasant category & -0.021 & NS & 0.013 & NS & 0.537 & $<0.01$ & 0.604 & $<0.01$ \\
\hline FQL-Frightening category & -0.350 & $<0.01$ & -0.485 & $<0.01$ & -0.169 & NS & -0.415 & $<0.01$ \\
\hline Dyspnea (mMRC) & -0.564 & $<0.01$ & -0.620 & $<0.01$ & -0.508 & $<0.01$ & -0.666 & $<0.01$ \\
\hline Depression (HADS-Depression) & -0.414 & $<0.01$ & -0.625 & $<0.01$ & -0.489 & $<0.01$ & -0.656 & $<0.01$ \\
\hline Age (years) & -0.157 & NS & 0.006 & NS & 0.026 & NS & -0.070 & NS \\
\hline Gender & -0.100 & NS & -0.012 & NS & -0.171 & NS & -0.297 & $<0.05$ \\
\hline Comorbidity frequency & -0.361 & $<0.01$ & -0.245 & NS & -0.223 & NS & -0.340 & $<0.01$ \\
\hline TLCO\%Pred & 0.417 & $<0.01$ & 0.488 & $<0.01$ & 0.168 & NS & 0.232 & NS \\
\hline
\end{tabular}

P, statistically significant; $\rho$, Spearman rank correlation coefficient. CIS-Fatigue, Checklist Individual Strength subscale Fatigue; EQ-5D-5L IV, EuroQol, 5 dimensions, Index Value; EQ-5D-5L VAS, EuroQol, 5 dimensions, Visual Analog Scale; FCS, Fatigue Catastrophizing Scale; FQL, Fatigue Quality Scale; HADS-Anxiety, Hospital Anxiety and Depression Scale subscale Anxiety; HADS-Depression, Hospital Anxiety and Depression Scale subscale Depression; mMRC, modified Medical Research Council-Dyspnea; VAS, Visual Analogue Scale; TLCO \%Pred, Transfer Factor of the lung for carbon monoxide (measured in $\mathrm{mL} / \mathrm{min} / \mathrm{mmHg}$ ) percentage predicted.

Table 6 Regression equations for general health (EQ-5D-5L VAS or EQ-5D-5L index value) in patients with idiopathic pulmonary fibrosis or sarcoidosis

\begin{tabular}{|c|c|c|c|c|}
\hline & Regression equation & $\mathrm{R}$ & $\mathrm{R}^{2}$ & SEE \\
\hline EQ-5D-5L VAS & $\begin{array}{l}80.700-0.529^{*} \mathrm{CIS}-\text { Fatigue }-1.611^{*} \text { HADS-Anxiety }-5.440^{*} \\
\text { Comorbidities }+0.339^{*} \text { TLCO } \% \text { Pred }\end{array}$ & 0.76 & 0.58 & 11.72 \\
\hline EQ-5D-5L index value & $\begin{array}{l}0.829-0.003^{\star} \text { FQL-Exhausted }-0.032^{*} \mathrm{mMRC}-0.011^{*} \mathrm{HADS}-\text { Anxiety }- \\
0.014^{\star} \mathrm{H} \text { ADS-Depression }+0.003^{*} \text { TLCO } \% \text { Pred }\end{array}$ & 0.87 & 0.75 & 0.10 \\
\hline EQ-5D-5L VAS & $\begin{array}{l}98.672-0.815^{\star} \mathrm{CIS}-\text { Fatigue }+0.340^{\star} \mathrm{FQL}-\text { Pleasant }-1.387^{\star} \mathrm{HADS}- \\
\text { Depression }\end{array}$ & 0.77 & 0.59 & 12.73 \\
\hline EQ-5D-5L index value & $0.986-0.002^{*} \mathrm{FQL}-$ Exhausted $-0.070^{*} \mathrm{mMRC}-0.019^{*} \mathrm{HADS}-$ Anxiety & 0.75 & 0.56 & 0.15 \\
\hline
\end{tabular}

Regression equations with backwards analysis, dependent variable EQ-5D-5L VAS or EQ-5D-5L index value, independent variables in univariate analyses significant $(\mathrm{P}<0.05)$ correlated; only equations are presented after the final backward regression analysis. CIS-Fatigue, Checklist Individual Strength subscale fatigue; EQ-5D-5L, EuroQol 5 dimensions 5 levels; FCS, Fatigue Catastrophizing Scale; FQLExhausted, Fatigue Quality List category exhausted; FQL-Pleasant, Fatigue Quality List category pleasant; HADS-Anxiety, Hospital Anxiety and Depression Scale subscale anxiety; HADS-Depression, Hospital Anxiety and Depression Scale subscale depression; IPF, idiopathic pulmonary fibrosis; mMRC, modified Medical Research Council dyspnea scale; TLCO \%Pred, Transfer Factor of the lung for carbon monoxide percentage predicted; VAS, Visual Analog Scale. 
of Bloem et al. [2020] showed a slight correlation between CIS-fatigue score and sleepiness (ESS $>10)$ in patients with sarcoidosis $(\rho=0.282 ; \mathrm{P}<0.05)$ but not in patients with IPF. In the search for different experiences of fatigue is sleepiness not included. Remarkable in the study of Bosse Henck (38) severe fatigue in patients with sarcoidosis was found in (only) $16 \%$ of the total sample while in the current study it is much higher (i.e., 69\%). Both studies used a different fatigue questionnaire, Bosse Henck used the Fatigue Assessment Scale (39-41) and current study used the CIS-Fatigue. The intracorrelation between the FAS and the CIS-Fatigue is to be expected seemly high. So differences in fatigue severity results is expected to be part of different sarcoidosis cohorts. The German cohort existed from members of the German Sarcoidosis Society in contrary of current study with a well-defined pulmonary sarcoidosis cohort.

A limitation of the current study is that all patients were recruited in an outpatient clinic of a respiratory medicine department. Therefore, these results may not be generalizable to patients with IPF or sarcoidosis in other settings. In addition, fatigued patients with negative perceptions of fatigue might not be willing to participate in a questionnaire-based fatigue study. Insights why patients were not responding have remained unknown due to inability to study non-participating patients. Strengths of the study are the cross-sectional design, the good distribution of patients between the non-severely and the severely fatigued groups and the relatively large sample size of the number of patients with IPF.

\section{Conclusions}

In conclusion, this is the first study investigating experiences of fatigue in patients with IPF or pulmonary sarcoidosis. Professionals treating patients with IPF or sarcoidosis not only should focus on fatigue severity, but also on the subjective experiences related to fatigue. In addition, not only severely fatigued patients may have negative fatiguerelated experiences, but also non-severely fatigued patient may have these. Although similarities were found in the connotations of fatigue across non-severe and severe fatigued patients, differences were also evident and could be mapped for IPF and sarcoidosis.

\section{Acknowledgments}

Funding: None.

\section{Footnote}

Reporting Checklist: The authors have completed the SURGE reporting checklist. Available at https://dx.doi. org/10.21037/jtd-21-462

Data Sharing Statement: Available at https://dx.doi. org/10.21037/jtd-21-462

Conflicts of Interest: All authors have completed the ICMJE uniform disclosure form (available at https://dx.doi. org/10.21037/jtd-21-462). MAS reports grants from Lung Foundation Netherlands, grants from Stichting Astma Bestrijding, grants and personal fees from Boehringer Ingelheim, and grants and personal fees from AstraZeneca, outside the submitted work. The other authors have no conflicts of interest to declare.

Ethical Statement: The authors are accountable for all aspects of the work in ensuring that questions related to the accuracy or integrity of any part of the work are appropriately investigated and resolved. The study was conducted in accordance with the Declaration of Helsinki (as revised in 2013). Medical Ethical Committee approval was granted (METCZ20180027) and registration with the Netherlands Trial Register (Code 7201) was established, written informed consent was obtained.

Open Access Statement: This is an Open Access article distributed in accordance with the Creative Commons Attribution-NonCommercial-NoDerivs 4.0 International License (CC BY-NC-ND 4.0), which permits the noncommercial replication and distribution of the article with the strict proviso that no changes or edits are made and the original work is properly cited (including links to both the formal publication through the relevant DOI and the license). See: https://creativecommons.org/licenses/by-nc-nd/4.0/.

\section{References}

1. Bradley B, Branley HM, Egan JJ, et al. Interstitial lung disease guideline: the British Thoracic Society in collaboration with the Thoracic Society of Australia and New Zealand and the Irish Thoracic Society. Thorax 2008;63 Suppl 5:v1-58.

2. Travis WD, Costabel U, Hansell DM, et al. An official American Thoracic Society/European Respiratory Society statement: Update of the international multidisciplinary 
classification of the idiopathic interstitial pneumonias. Am J Respir Crit Care Med 2013;188:733-48.

3. U.S. Food and Drug Administration's. A series of reports from the U.S. Food and Drug Administration's (FDA's) Patient-Focused Drug Development Initiative Idiopathic Pulmonary Fibrosis Public Meeting: September 26, 2014. Available online: https://www.fda.gov/downloads/ ForIndustry/UserFees/PrescriptionDrugUserFee/ UCM440829.pdf

4. Korenromp IHE, Heijnen CJ, Vogels OJM, et al. Characterization of chronic fatigue in patients with sarcoidosis in clinical remission. Chest 2011;140:441-7.

5. Voortman M, Hendriks CMR, Elfferich MDP, et al. The Burden of Sarcoidosis Symptoms from a Patient Perspective. Lung 2019;197:155-61.

6. Bloem AEM, Mostard RLM, Stoot N, et al. Severe Fatigue is Highly Prevalent in Patients with IPF or Sarcoidosis. J Clin Med 2020;9:1178.

7. Gielissen MF, Knoop H, Servaes P, et al. Differences in the experience of fatigue in patients and healthy controls: patients' descriptions. Health Qual Life Outcomes 2007;5:36.

8. van Hoogmoed D, Fransen J, Bleijenberg G, et al. Physical and psychosocial correlates of severe fatigue in rheumatoid arthritis. Rheumatology (Oxford) 2010;49:1294-302.

9. Jaime-Lara RB, Koons BC, Matura LA, et al. A Qualitative Metasynthesis of the Experience of Fatigue Across Five Chronic Conditions. J Pain Symptom Manage 2020;59:1320-43.

10. Whitehead LC, Unahi K, Burrell B, et al. The Experience of Fatigue Across Long-Term Conditions: A Qualitative Meta-Synthesis. J Pain Symptom Manage 2016;52:131-43.e1.

11. Raghu G, Collard HR, Egan JJ, et al. An official ATS/ ERS/JRS/ALAT statement: idiopathic pulmonary fibrosis: evidence-based guidelines for diagnosis and management. Am J Respir Crit Care Med 2011;183:788-824.

12. Hunninghake GW, Costabel U, Ando M, et al. ATS/ERS/ WASOG statement on sarcoidosis. American Thoracic Society/European Respiratory Society/World Association of Sarcoidosis and other Granulomatous Disorders. Sarcoidosis Vasc Diffuse Lung Dis 1999;16:149-73.

13. Zhou Y, Lower EE, Li H, et al. Clinical management of pulmonary sarcoidosis. Expert Rev Respir Med 2016;10:577-91.

14. Quanjer PH, Tammeling GJ, Cotes JE, et al. Lung volumes and forced ventilatory flows. Work Group on Standardization of Respiratory Function Tests. European
Community for Coal and Steel. Official position of the European Respiratory Society. Rev Mal Respir 1994;11 Suppl 3:5-40.

15. Vercoulen JH, Swanink CM, Fennis JF, et al. Dimensional assessment of chronic fatigue syndrome. J Psychosom Res 1994;38:383-92.

16. Beurskens AJ, Bültmann U, Kant I, et al. Fatigue among working people: validity of a questionnaire measure. Occup Environ Med 2000;57:353-7.

17. Bültmann U, de Vries M, Beurskens AJ, et al. Measurement of prolonged fatigue in the working population: determination of a cutoff point for the checklist individual strength. J Occup Health Psychol 2000;5:411-6.

18. Worm-Smeitink M, Gielissen M, Bloot L, et al. The assessment of fatigue: Psychometric qualities and norms for the Checklist individual strength. J Psychosom Res 2017;98:40-6.

19. Bestall JC, Paul EA, Garrod R, et al. Usefulness of the Medical Research Council (MRC) dyspnoea scale as a measure of disability in patients with chronic obstructive pulmonary disease. Thorax 1999;54:581-6.

20. Sullivan MJ, Bishop SR, Pivik J. The pain catastrophizing scale: development and validation. Psychol Assess 1995;7:524.

21. Sullivan MJL. PCS: Pain Catastrophizing Scale: user manual. Montreal: Departments of Psychology, Medicine, and Neurology, School of Physical and Occupational Therapy. McGill University; 2009.

22. Zigmond AS, Snaith RP. The hospital anxiety and depression scale. Acta Psychiatr Scand 1983;67:361-70.

23. Cosco TD, Doyle F, Ward M, et al. Latent structure of the Hospital Anxiety And Depression Scale: a 10-year systematic review. J Psychosom Res 2012;72:180-4.

24. Herdman M, Gudex C, Lloyd A, et al. Development and preliminary testing of the new five-level version of EQ-5D (EQ-5D-5L). Qual Life Res 2011;20:1727-36.

25. van Reenen M, Janssen B. EQ-5D-5L user guide: basic information on how to use the EQ-5D-5L instrument. Rotterdam: EuroQol Research Foundation, 2015.

26. Altman DG. Practical statistics for medical research. Lyon: CRC Press; 1990.

27. Akinwande MO, Dikko HG, Samson A. Variance inflation factor: as a condition for the inclusion of suppressor variable (s) in regression analysis. Open Journal of Statistics 2015;5:754.

28. Greenberger D, Padesky CA. Mind over Mood: a cognitive therapy treatment manual for clients. New York: Guilford Press; 1995. 
29. Thoma N, Pilecki B, McKay D. Contemporary Cognitive Behavior Therapy: A Review of Theory, History, and Evidence. Psychodyn Psychiatry 2015;43:423-61.

30. Moor CC, Kahlmann V, Culver DA, et al. Comprehensive Care for Patients with Sarcoidosis. J Clin Med 2020;9:390.

31. Saketkoo LA, Karpinski A, Young J, et al. Feasibility, utility and symptom impact of modified mindfulness training in sarcoidosis. ERJ Open Res 2018;4:00085-2017.

32. Poort H, Peters MEWJ, van der Graaf WTA, et al. Cognitive behavioral therapy or graded exercise therapy compared with usual care for severe fatigue in patients with advanced cancer during treatment: a randomized controlled trial. Ann Oncol 2020;31:115-22.

33. White PD, Goldsmith KA, Johnson AL, et al. Comparison of adaptive pacing therapy, cognitive behaviour therapy, graded exercise therapy, and specialist medical care for chronic fatigue syndrome (PACE): a randomised trial. Lancet 2011;377:823-36.

34. van den Akker LE, Beckerman H, Collette EH, et al. Effectiveness of cognitive behavioral therapy for the treatment of fatigue in patients with multiple sclerosis: A systematic review and meta-analysis. J Psychosom Res 2016;90:33-42.

Cite this article as: Bloem AEM, Mostard RLM, Stoot N, Vercoulen JH, Peters JB, Spruit MA. Perceptions of fatigue in patients with idiopathic pulmonary fibrosis or sarcoidosis. J Thorac Dis 2021;13(8):4872-4884. doi: 10.21037/jtd-21-462
35. de Kleijn WP, Drent M, De Vries J. Nature of fatigue moderates depressive symptoms and anxiety in sarcoidosis. Br J Health Psychol 2013;18:439-52.

36. Luk EK, Gorelik A, Irving L, et al. Effectiveness of cognitive behavioural therapy in a community-based pulmonary rehabilitation programme: A controlled clinical trial. J Rehabil Med 2017;49:264-9.

37. Corfield EC, Martin NG, Nyholt DR. Co-occurrence and symptomatology of fatigue and depression. Compr Psychiatry 2016;71:1-10.

38. Bosse-Henck A, Koch R, Wirtz H, et al. Fatigue and Excessive Daytime Sleepiness in Sarcoidosis: Prevalence, Predictors, and Relationships between the Two Symptoms. Respiration 2017;94:186-97.

39. Michielsen HJ, De Vries J, Van Heck GL. Psychometric qualities of a brief self-rated fatigue measure: The Fatigue Assessment Scale. J Psychosom Res 2003;54:345-52.

40. Michielsen HJ, De Vries J, Van Heck GL, et al. Examination of the dimensionality of fatigue. Eur J Psychol Assess 2004;20:39-48.

41. De Vries J, Michielsen H, Van Heck GL, et al. Measuring fatigue in sarcoidosis: the Fatigue Assessment Scale (FAS). Br J Health Psychol 2004;9:279-91. 